\title{
POINTS OF MINIMUM NORM ON SMOOTH SURFACES IN BANACH SPACES
}

\author{
ROBERT BONIC ${ }^{1}$
}

Theorem. Suppose E is a real Banach space, and $\phi$ is a continuously Fréchet differentiable real valued function defined on E. Assume that for some $c$ in $R$ there is a $u_{0}$ in $\phi^{-1}(c)$ such that $\left\|u_{0}\right\| \leqq\|u\|$ for all $u$ in $\phi^{-1}(c)$. Then $\left|\phi^{\prime}\left(u_{0}\right) \cdot u_{0}\right|=\left\|\phi^{\prime}\left(u_{0}\right)\right\|\left\|u_{0}\right\|$.

Proof. We may assume that $u_{0}$ and $\phi^{\prime}\left(u_{0}\right)$ are not zero. Let $K=\operatorname{Ker} \phi^{\prime}\left(u_{0}\right)$. It will first be shown that $k \in K$ implies $\left\|u_{0}+k\right\|$ $\geqq\left\|u_{0}\right\|$.

Choose $u_{1} \in E$ with $\phi^{\prime}\left(u_{0}\right) \cdot u_{1}=1$ and let $E_{2}=\operatorname{span}\left\{u_{1}\right\}$. Then $E=K \times E_{2}$ and any $u \in E$ can be written $u=\left(y, \alpha u_{1}\right)$ where $y \in K$ and $\alpha=\phi^{\prime}\left(u_{0}\right) \cdot u$. Let $u_{0}=\left(y_{0}, \alpha_{0} u_{1}\right)$. Since $\phi^{\prime}\left(u_{0}\right) \neq 0$ we may apply the implicit function theorem (see [2]) to obtain a $C^{1}$-function $g: U_{1} \rightarrow R$ where $U_{1}$ is a convex open neighborhood of zero in $K$ and $g$ satisfies $g(0)=0, g^{\prime}(0)=0$ and

$$
\phi\left(y_{0}+h, \alpha_{0} u_{1}+g(h) u_{1}\right)=c \quad \text { for all } h \in U_{1} .
$$

Let $B=\left\{u \in E:\|u\|<\left\|u_{0}\right\|\right\}$. By assumption $\phi^{-1}(c) \cap B=\varnothing$. Assuming $\left(u_{0}+K\right) \cap B \neq \varnothing$ we will obtain a contradiction. Suppose there is a $k \in K$ with $\left\|u_{0}+k\right\|<\left\|u_{0}\right\|$. We may assume that $k \in U_{1}$ and $g(t k)>0$ for $0<t \leqq 1$. Then for some $s$ with $0<s<1$ we have that $\left(y_{0}+k, \alpha_{0} u_{1}+s g(k)\right) \in B$. Since $B$ is convex $\left(y_{0}+\sigma k, \alpha_{0} u_{1}+\sigma s g(k)\right) \in B$ for $0<\sigma \leqq 1$ so $g(\sigma k) \geqq \sigma s g(k)$ for $0<\sigma \leqq 1$. Therefore $(g(\sigma k)-g(0)) / \sigma$ $\geqq \sigma s g(h) / \sigma=s g(k) \neq 0$. In other words $g^{\prime}(0) \cdot k \neq 0$ and this is a contradiction.

Therefore $\left\|u_{0}+k\right\| \geqq\left\|u_{0}\right\|$ for all $k \in K$, and it follows that $u_{0} \notin K$ and $\left\|\alpha u_{0}+k\right\| \geqq\left\|\alpha u_{0}\right\|$ for all $\alpha \in R$ and $k \in K$. Let $\epsilon>0$ and choose $v \in E$ with $\|v\|=1$ and $\left|\phi^{\prime}\left(u_{0}\right) \cdot v\right| \geqq\left\|\phi^{\prime}\left(u_{0}\right)\right\|-\epsilon$. Then $v=\alpha u_{0}+k$, $k \in K$ and we have that $1=\left\|\alpha u_{0}+k\right\| \geqq|\alpha|\left\|u_{0}\right\|$. Hence $\left\|\phi^{\prime}\left(u_{0}\right)\right\|-\epsilon$ $\leqq\left|\phi^{\prime}\left(u_{0}\right) \cdot v\right|=\left|\phi^{\prime}\left(u_{0}\right) \cdot \alpha u_{0}\right| \leqq\left|\phi^{\prime}\left(u_{0}\right) \cdot u_{0}\right| /\left\|u_{0}\right\|, \quad$ so $\left\|\phi^{\prime}\left(u_{0}\right)\right\|\left\|u_{0}\right\|$ $-\epsilon|| u_{0} \| \leqq\left|\phi^{\prime}\left(u_{0}\right) \cdot u_{0}\right|$. Since $\epsilon$ is arbitrary $\left\|\phi^{\prime}\left(u_{0}\right)\right\|\left\|u_{0}\right\| \leqq\left|\phi^{\prime}\left(u_{0}\right) \cdot u_{0}\right|$. The reverse inequality is trivial, so the proof is complete.

Corollary 1. Let $\phi, E$, and $u_{0}$ be as in the theorem. Suppose $E=F^{*}$ where $F$ is separable and $\phi\left(u_{n}\right) \rightarrow \phi\left(u_{0}\right)$ whenever $u_{n} \cdot x \rightarrow u_{0} \cdot x$ for all $x \in E$. Then in addition to the above conclusion it follows that $\phi^{\prime}\left(u_{0}\right) \in F$.

Received by the editors December 5, 1966.

${ }^{1}$ Research supported in part by NSF grant GP 5619. 
Proof. $\phi^{\prime}\left(u_{0}\right) \in F^{* *}$ and to show that $\phi^{\prime}\left(u_{0}\right) \in F$ it is sufficient to show that $\phi^{\prime}\left(u_{0}\right) \cdot u_{n} \rightarrow 0$ whenever $u_{n} \cdot x \rightarrow 0$ for all $x \in F$. Assuming this is false we can find a sequence $\left\{v_{n}\right\} \subset F^{*}$ with $\left\|v_{n}\right\|=1$ and $\left|\phi^{\prime}\left(u_{0}\right) \cdot v_{n}\right|>\gamma>0$ for all $n$ and some $\gamma>0$. Let

$$
\alpha_{n}=\max \left\{\left|\phi\left(u_{0}+t v_{n}\right)-\phi\left(u_{0}\right)\right|: 0 \leqq t \leqq 1\right\} .
$$

Then $\alpha_{n} \rightarrow 0$ and we have that $\left|\left(\phi\left(u_{0}+\beta_{n} v_{n}\right)-\phi\left(u_{0}\right)\right) / \beta_{n}\right| \leqq \alpha_{n} / \beta_{n} \rightarrow 0$ where $\beta_{n}=\alpha_{n}^{1 / 2}$. However

$$
\left|\phi\left(u_{0}+v\right)-\phi\left(u_{0}\right)-\phi^{\prime}\left(u_{0}\right) \cdot v\right| /\|v\| \rightarrow 0 \quad \text { as }\|v\| \rightarrow 0
$$

so that $\left|\left(\left(\phi\left(u_{0}+\beta_{n} v_{n}\right)-\phi\left(u_{0}\right)\right) / \beta_{n}\right)-\left(\phi^{\prime}\left(u_{0}\right) \cdot v_{n}\right)\right| \rightarrow 0$ as $n \rightarrow \infty$ giving that $\left|\phi^{\prime}\left(u_{0}\right) \cdot v_{n}\right| \rightarrow 0$ which is a contradiction.

Corollary 2. Assume the hypothesis of the above corollary and suppose that $F=L^{1}[0,1]$ so $F^{*}=L^{\infty}[0,1]$. Then $u_{0} \in L^{\infty}, \phi^{\prime}\left(u_{0}\right) \in L^{1}$ and we have that $u_{0}(t)= \pm\left\|u_{0}\right\| \operatorname{sgn} \phi^{\prime}\left(u_{0}\right)(t)$ almost everywhere where $\phi^{\prime}\left(u_{0}\right)(t) \neq 0$. In particular, if $\phi^{\prime}\left(u_{0}\right)(t) \neq 0$ almost everywhere, $u_{0}$ is a "bang-bang" type solution which often occurs in control theory.

REMARKs. (a) The conclusion above may be phrased in another way. Namely, $\phi^{\prime}\left(u_{0}\right) /\left\|\phi^{\prime}\left(u_{0}\right)\right\|$ is a support functional to the unit sphere in $E$ at the point $\pm u_{0}\left\|u_{0}\right\|$. If the norm $N(u)=\|u\|$ is differentiable (except at zero) then support functionals are unique and it follows that $N^{\prime}\left(u_{0}\right)= \pm \phi^{\prime}\left(u_{0}\right)$. In this case the above theorem reduces to the Lagrange method of multiplier result.

(b) Corollary 2 is proved in [3, pp. 302-311], for a special class of constraints $\phi$. The result there suggested the above theorem. In [3] the argument is basically the following: First extend $\phi$ to $L^{p}[0,1]$, $(1<p<\infty)$. Then, since the norm in $L^{p}$ is differentiable, a Lagrange multiplier argument applies to give a solution $u_{p}$. $u_{0}$ is obtained by letting $p \rightarrow \infty$.

The norm in $L^{\infty}[0,1]$ is nowhere differentiable, and in fact cannot be approximated by a differentiable function [1]. Therefore in using a Lagrange multiplier argument in [3], the indirect approach via $L^{p}$ was essential.

\section{BIBLIOGRAPHY}

1. R. Bonic and J. Frampton, Differentiable functions on certain Banach spaces, Bull. Amer. Math. Soc. 71 (1965), 393-395.

2. S. Lang, Introduction to differentiable manifolds, Interscience, New York, 1962.

3. T. Saaty and J. Bram, Nonlinear mathematics, McGraw-Hill New York, 1964.

NORTHEASTERN UNIVERSITY 